\title{
Plant chemical ecology finally gets to its root(s)
}

Nicole M. van Dam, Radboud University Nijmegen, Ecogenomics, Institute of Water and Wetland Research (IWWR), Heyendaalseweg 135, 6525 AJ Nijmegen, The Netherlands ${ }^{1}$

Plants produce a wide array of secondary compounds that play critical roles in the interaction with their dynamic environment. Since the first issues of the Journal of Chemical Ecology in 1974, numerous papers have been published detailing the function of volatile and non-volatile plant compounds in interactions between plants, herbivores and their natural enemies. Initially, plant chemical-ecological studies focused mainly on aboveground interactions. One can only speculate about what caused this focus; most likely because it is so much easier to work on organisms and plant organs that one can see. However, roots have a 'life of their own' below the ground and may have as many, or even more, interactions with other organisms as the shoots. It has been known for centuries that plant roots contain high levels of medicinal compounds. A famous example is the roots of the legendary mandrake (Mandragora officinale), whose toxic and hallucinatory compounds have been used to concoct witchcraft potions as well as to kill opponents. Moreover, many well-studied defense compounds, such as pyrrolizidine alkaloids in common ragwort (Jacobaea vulgaris) and nicotine in (wild) tobacco, are exclusively produced in the roots before they are transported aboveground. For some reason, however, this was not interpreted as a sign that roots might need those chemicals as protection against herbivores and other enemies as well. Actually, roots generally were considered to be less prone to attack because they were assumed to be less apparent to herbivores.

The tide has turned for root chemical ecology. A short survey in ISI Web of Knowledge identified 140 records from JCE on the chemical ecology of plant roots. ${ }^{2}$ About one third (47) of these papers were on allelopathic effects, whereas 43 reported on root-herbivore interactions. Induced defenses were studied in 21 of these records, half of which were published in the last seven years. This also is a global trend when looking at the distribution of published records over time: from 1983-1996 ${ }^{3}$ only two papers on root chemical ecology were published each year, a number that rose to five between 1997 and 2007, and has increased ever since to a steady eight papers per year between 1997 and 2011. In June 2012, a special issue focused on soil chemical ecology and last year, we saw an all- time record of 21 papers, undoubtedly due to the special issues on allelochemicals in natural and agro-ecosystems and plantmicrobe interactions published in January and July 2013, respectively. In my opinion, these numerical data clearly illustrate the point that root chemical ecology is on the rise, not only in the JCE but also in other publications.

Now that roots are on the research agenda, it is time to look forward and identify where we should go. Luckily, we do not have to 'grope in the dark'. The extensive body of aboveground research provides us with clear concepts that can guide belowground research efforts. For example, optimal defense allocation theory predicts that those parts that are the most valuable to the plant and/or the most vulnerable to attack, should be the best protected. Indeed, in aboveground plant organs, it generally is observed that seeds and young leaves have higher levels of chemical defenses than senescing leaves, which are less valuable and palatable to herbivores. If roots are included in these analyses at all, they

\footnotetext{
${ }^{1}$ Many thanks to J. Daniel Hare, University of California, Riverside, for valuable comments on an earlier version of this essay.

2 ISI WoS search on 17 January 2014, search terms "root" OR "roots" AND "Journal of Chemical Ecology" (382 records). Records solely reporting on sex pheromones of root pests, root growth effects (of isolated or synthetic compounds) were manually removed.

${ }^{3}$ No records with the above search terms were retrieved from earlier issues. This may have several reasons: JCE $1974-1976$ are not indexed in WoS and abstracts are not available for the older issues. However, a quick scan of the JCE issues of that time revealed that in those year the papers focused mainly on sex pheromones and kairomone research.
} 
are seldom subdivided into different (functional) classes. This is not just, because losing a few fine roots to a herbivore may have less dramatic fitness effects than damage to the main root. In the latter case, herbivore feeding can cause a disruption of water transport to the shoot as well as assimilate transport to the roots, which may have fatal consequences for the plant as a whole. Therefore, it may be expected that main roots contain the highest levels of chemical defenses. On a finer scale, the allocation of chemical defenses may be spatially explicit. Defenses aimed at chewing herbivores and pathogens may be allocated to peripheral tissues, the epidermis and the cortex, whereas defenses against suckingpiercing endopathogenic nematodes may be concentrated in the endodermis and the central cylinder. It also should be recognized that shoots and roots are living in a completely different environments. As a consequence, the types of defense compound in both organs may differ completely. For example, May Berenbaum and Art Zangerl showed that furanocoumarins are prominent defense compounds in parsnip (Pastinaca sativa) plants. The concentrations of these furanocoumarins are the highest in flowers and seeds, whereas the concentrations in the roots were very low. At the time, the authors postulated that roots do not need such high levels, as they have a lower chance of being attacked. However, it is more likely that furanocoumarins, which are potentiated when exposed to UV light, are quite harmless - and thus useless as anti-herbivore defenses - in the darkness of the soil. Similarly, specific glucosinolates are more prominent in roots than in shoots of the same plant. This may be related to the fact that the toxic breakdown products of these specific root glucosinolates, the isothiocyanates, have chemical properties that make them more suitable for transport in the soil. Similar questions pertain to the large and chemically diverse class of terpenoids, which are produced by both roots and shoots upon insect attack. On the one hand, it may be more difficult for non-polar terpenoids to travel through humid soils. On the other hand, there is a lower chance of breakdown of such compounds due to high UV or ozone levels. It is as yet to be assessed whether the types of terpenoids produced by roots and shoots show signatures of optimization for biological activity in soil or air.

Finally, it still is not known if roots have the same perception and signaling mechanisms for herbivoreinduced responses defenses as shoots. Using feral cabbages, we have shown that application of jasmonic acid, the signaling hormone involved in herbivore-induced responses, has a distinctly different effect on plant volatile and glucosinolate levels when added to the roots. Roots thus respond differently to jasmonic acid, possibly because their lipoxygenase pathway is organized in a different way. Possibly, roots work with other amino-acid conjugates than jasmonic acid-isoleucine, which is the active form triggering herbivore-induced responses in the shoots. Certainly, it shows that the optimization of root and shoot defenses can follow differential evolutionary trajectories even within one integrated organism.

Research on the chemical ecology of roots will benefit greatly from recent developments in chemicalanalytical technology. For example, Proton- Transfer Mass Spectrometry can measure the production of root volatiles in real time at a sensitivity that is close to the sensitivity of insect antennae.

Metabolomics, combined with transcriptomic analyses, will allow us to see the full complement of herbivore-induced changes in plants instead of only 'the usual suspects' analyzed by targeted analyses. At the same time, genomic technologies such as metagenomics or cDNA barcoding of soil and gut microorganisms, will provide more insight into potential belowground interactors. The main challenge will be to identify which ones, among these many compounds, genes, and microbes, are the most important regarding the interaction between the plant and its environment. This knowledge can be obtained only by experimentally manipulating specific compounds or interactors in the soil, and measuring the consequences thereof in terms of plant fitness.

Certainly, the original challenges associated with root research will remain: it is hard to see what is going on when manipulating roots and belowground herbivores. However, the perseverant scientist that will take the challenge undoubtedly will gain the satisfaction of unearthing many new discoveries that lay waiting for us in this 'terra incognita'. 


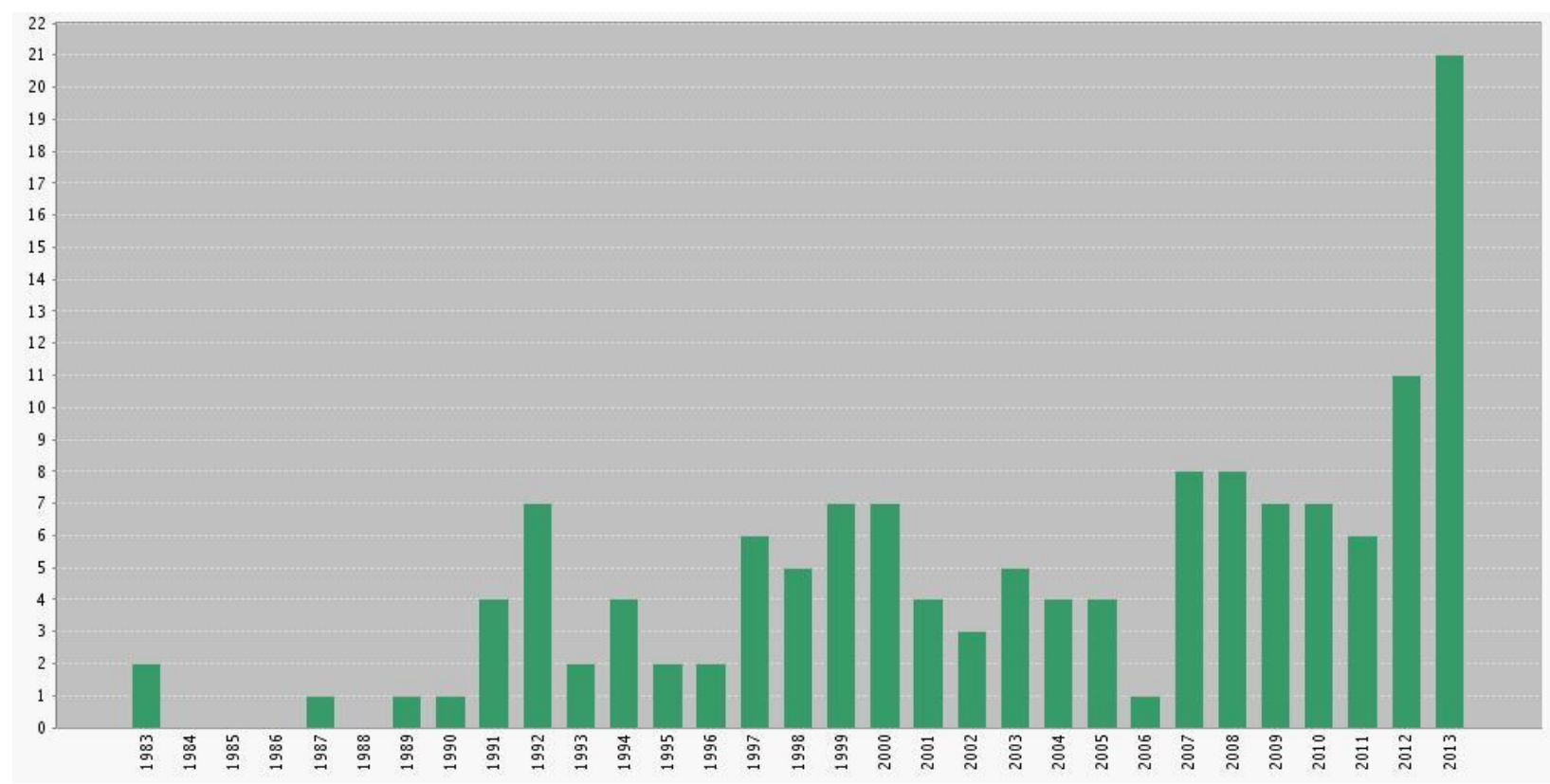

JCE: Root chemical ecology papers 1983 to 2013. Source: ISI WoS

(Thomson Reuters). 